\title{
OTO - A DIY Platform for Mobile Social Robots in Education
}

\author{
Thomas Vervisch ${ }^{1}$, Natan Doms ${ }^{1}$, Sander Descamps ${ }^{2}$, Cesar Vandevelde ${ }^{1}$, \\ Francis wyffels ${ }^{2}$, Steven Verstockt ${ }^{2}$, and Jelle Saldien ${ }^{1}$ \\ 1 Ghent University, Department of Industrial Systems and Product Design, \\ \{natan.doms, tbvervis.vervisch, sander.descamps, cesar.vandevelde, \\ jelle.saldien\}@ugent.be \\ 2 Ghent University, Department of Electronics and Information Systems, \\ \{francis.wyffels, steven.verstockt\}@ugent.be
}

\begin{abstract}
This paper describes the design of OTO, a do-it-yourself expansion kit for OPSORO (Open Platform for Social Robots), that enables and facilitates the creation of mobile social robots. The expansion kit consists of modular, adaptable building blocks combined with a software toolkit, and is aimed at applications within the maker community, STEM education, and the market for creative inventor kits. Keeping reproducibility and adaptability in mind, the expansion kit can be produced entirely using digital manufacturing technology and low-cost, offthe-shelf components. Using the building blocks offered by this system, users can easily design, build and customize mobile social robots. The software is designed to address a wide range of users by offering different programming options depending on the user's skill and experience. Inexperienced users are offered a graphical programming environment based on Blockly, whereas more advanced users can program their robot using Lua or Python. The OTO toolkit offers a fun and playful context in which a wide range of STEM-related skills are addressed.
\end{abstract}

Keywords: social robotics, human-robot interaction, emotions, facial expressions, mechatronics, DIY, modularity

\section{Introduction}

Recent years have been marked by a rise of digital rapid prototyping techniques [6], such as lasercutting and 3D printing. Access to these technologies has also steadily been improved through FabLabs [8], the huge amount of web resources, open source projects $[4,7]$, and the support of the "maker movement" [2]. This has resulted in increased possibilities for makers to build their own custom robots [11]. Despite this evolution, there was a lack of open-source and easily adaptable social robotic platforms for human-robot interaction [3].

This has led to the development of OPSORO - Open Platform for Social Robotics [10] - a modular platform that enables human-robot interaction with do-it-yourself (DIY) social robots. This platform uses an innovative modular 
system design that enables the creation of different embodiments to represent anthropomorphic robots, emphasizing face-to-face communication. However, this platform is limited to stationary robots with facial expressions. This paper describes the process of adapting and extending this platform to enable mobile social robots. The expansion kit, OTO, is complementary with the existing OPSORO platform and has a similar design architecture. The mobile platform consists of modular units that transform a stationary social robot into a vehicle-like robot with facial expressions, similar to cartoon characters (e.g. "Cars" and "Thomas the Train"). Furthermore, OTO has been designed with modular subunits built from off-the-shelf and easy-to-produce components. In this way, users can build and customize different embodiements of social robots. For programming the OPSORO offers multiple options to address a wide range of users with varying programming skills.

\section{Application in Educational Settings}

The topic of robotics is frequently chosen by teachers as the subject of STEMfocused problem-based learning. The reason for this is obvious: as Benitti [1] and Johnson [5] show, teaching robotics is a very effective way to motivate students and integrates many different knowledge domains of the curriculum. Furthermore, it also stimulates students' social and teamwork skills. As a secondary aspect, robots are something that captures the imagination of many children, which serves as a motivator [5].

Research shows that the design of the robot can improve social HRI in the correct context [9]. Furthermore, the link between the development of a meaningful social interaction between robots and people and the degree of anthropomorphism in the physical characteristics of a robot has been investigated and shows us that this anthropomorphism has a positive impact if used correctly [3].

\section{Design of the OPSORO Hardware}

A rolling platform was chosen to make the social robot mobile. This solution offers a higher efficiency than a walking robot, allowing longer run times with batteries [13]. In addition, the robot does not have to be connected through a tether, allowing it to move freely. A rolling platform also minimizes the complexity of a mobile robot, facilitating the construction process for less experienced users. Differential steering was chosen as the steering method. This method minimizes the mechanical complexity of the robot, thus limiting the number of modules needed. Differential steering makes the robot more maneuverable by enabling it to rotate around its own axis.

As base plates to mount the emotion and driving modules, the same grid as the existing OPSORO kit is used, though with different dimensions. In order to be able to build up modules lengthwise, such as the driving modules, and in the height, such as the emotion modules, grids mounted perpendicular to each other are used instead of stacking the modules on one another. This enables a 
larger degree of variation in the positioning of the modules, both in height and in depth. The modules are designed in such a way that they can be fabricated from $3 \mathrm{~mm}$ ABS sheets using a lasercutter.

The modules are designed in such a way that they can be mounted on every face of the module. This increases the creative possibilities, which is an important aspect of the open source platform. Due to the differential steering, only one type of wheel module is required. The wheel-modules use continuous rotation servos. These servos do not have a limited travel angle and can therefore rotate continuously forward or backwards. They can be controlled in the same manner as regular servos, using the same hardware and software, which makes them ideal for this purpose. The mounted wheels consist of laser-cut slices onto which a K'Nex tire is attached. The slices are in turn attached to the servo horn. For aesthetic purposes, 3D printed or even cardboard wheel caps can also be attached.

The camera module is used as the extra input source for the social robot. It allows the robot to detect and react to events that occur in its environment. Facedetection and tracking algorithms were also implemented within the OPSORO platform, opening new modes of interaction. For example, the robot can say 'hello' when a a face is detected, or the robot can make eye contact when it detects someone. A Raspberry Pi provides the necessary computational power to complete these tasks.

The power source is an important aspect in order to make the robot completely mobile. The power supply issue of a mobile social robot is solved by using rechargeable NiMH batteries. These batteries provide sufficient power and are resistant to peak currents caused by initializing the servos. These off-the-shelf batteries are safe to use by children and other inexperienced users.

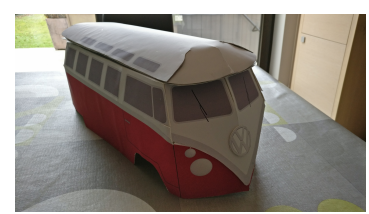

(a) Papercraft Volkswagen van

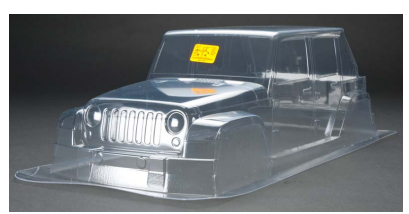

(b) RC car body

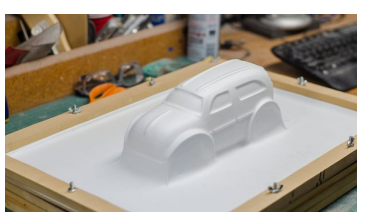

(c) Thermoform

Fig. 1. Possible car shells

We propose two options for the bodywork of vehicle-like social robots: thermoformed plastic shells and papercraft bodies. A papercraft body is a quick, easy and cheap way to create a one-of-a-kind social robot body. It can be made to any desired size, shape or sort of vehicle. This kind of body is ideal for workshops or for users who want to create a lot of different characters. A Volkswagen van prototype, shown in figure 1(a), was made to demonstrate the possibilities of this technique. 
The papercraft technique can also be used to create a quick mock-up version of a proposed thermoformed body. Keeping with the open source paradigm, templates of these papercraft bodies can also be easily shared online.

For the thermoformed bodies, there are again two possibilities: either a hobby-grade RC car body (figure 1(b)) can be purchased, or a thermoformed body (figure 1(c)) can be crafted using a DIY CNC-milled mold. The thermoformed bodies can be decorated according to personal taste. Erasable whiteboard markers can even be used for temporary decoration.

\section{Software Architecture}

The existing platform code is implemented as a web service running on a Raspberry Pi. The platform provides a simple web interface for the users. The web service offers a variety of apps, each of them having their own purpose. These apps have direct access to the back-end of the platform, which is a virtual module structure that corresponds to the physical building blocks of the robot. An schematic overview of the architecture is given in figure 2 . In order to implement the new functionalities in the existing OPSORO-platform, a number of changes were made in the software code.

To make the OTO ride, wheel modules were added into the structure. In the OPSORO platform, each physical building block corresponds to a module in code, and each module has to be configured to make the robot work. Because of the modularity of the building blocks, it is important to mirror that modularity in software. After the wheel modules were added to the software, the challenge arose to find a clear and intuitive way to configure and steer the robot.

By adding Entities and Groups, this issue was resolved. The underlying data structure was reorganized as a tree instead of a list of modules. Each Entity represents a node in this tree of modules. Groups are virtual sets of modules, they allow a single instruction to be executed on each module of that group. To further refine which entity in the data tree to control, a module tag system was introduced. These tags are based on the position and type of the entity (e.g.: [eye, right], [eye, left]), and offer a simple way to filter through all modules.

A final architecture change is the action system. Normally, the position of each servo is set individually through a function call. However, this system posed difficulties for children as it can be hard to know which servo needs to be set at which value to perform a certain movement. The action system was designed to provide a simpler way to execute certain movements. Users specify the tags of the components they wish to drive and the action they wish to execute, and the code does the rest. The software selects the right modules and searches for the correct method to call, making it easier for children to program their robot.

By adding a camera, extra possibilities for interaction between the robot and humans were introduced. A face detection and tracking algorithm makes it possible to detect human faces and follow them. The Viola-Jones algorithm [12] is used for face detection. In order to guarantee the necessary speed and stability 


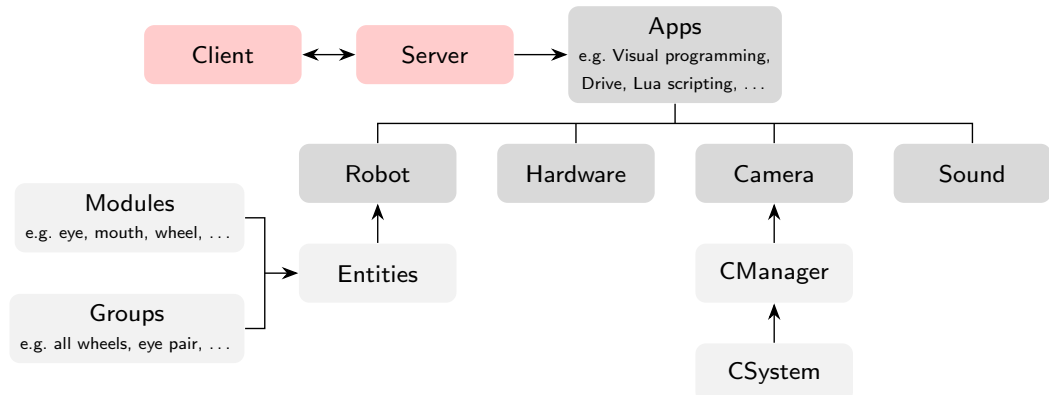

Fig. 2. Schematic overview of the software architecture

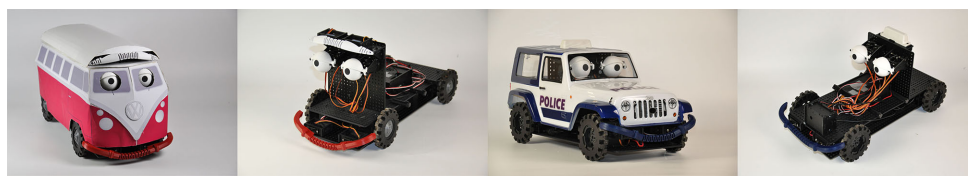

Fig. 3. Two DIY mobile social robots created using the OTO platform.

of the platform, the image processing algorithm is executed in a separate thread. This way, the computer vision tasks will not slow down the rest of the robot.

\section{Conclusion and Future Work}

In this paper, the design of a new social robot platform, OTO, is described. This social robot distinguishes itself from other social robots by providing an open source and modular building kit, allowing the user to fully customize their robot. OTO builds upon the existing OPSORO platform, but distinguishes itself by providing a mobility aspect and by linking emotion expressiveness with mobility. The software platform is easily accessible through a web interface and can be programmed using visual building blocks, opening new opportunities for children to build and interact with their robots.

The added dimension of mobility introduces many new possibilities for the OPSORO platform. It can be used to add new emotional behavior or to enable new functionality. The sensing capabilities of stationary robots are limited because they can only perceive what is nearby. By adding a mobile aspect to the platform, sensing capabilities are greatly expanded. For instance, the robot can follow a person or object, and can continuously gather new input from its environment. This input can be used to generate new behavioral or emotional outputs, as specified by the users' scripts.

The platform emphasizes a user-friendly interface that allows children and inexperienced programmers to use it. Still, the system is sufficiently flexible so that system developers and researchers can adapt it to specific tests and experiments. Because of the modular design, the platform can be easily expanded 
with new sensors and actuator modules. For these modules, new software blocks can also be developed to make them effortless to operated. In addition, due to the flexible and unspecified structure of the robot, new aesthetics (papercraft body, plastic shells, LEGO blocks, ... ) can be designed and shared. Finally, the research was implemented in two working prototypes, as shown in figure 3.

\section{References}

1. Benitti, F.B.V.: Exploring the educational potential of robotics in schools: A systematic review. Computers \& Education 58(3), 978-988 (apr 2012)

2. Chorianopoulos, K., Jaccheri, L., Nossum, A.S.: Creative and open software engineering practices and tools in maker community projects. Proceedings of the 4th ACM SIGCHI symposium on Engineering interactive computing systems - EICS '12 (January), 333 (2012)

3. Duffy, B.R.: Anthropomorphism and the social robot. In: Robotics and Autonomous Systems. vol. 42, pp. 177-190 (2003)

4. Hars, A., Ou, S.: Working for free? Motivations of participating in open source projects. International Journal of Electronic Commerce 6(3), 25-39 (2002)

5. Johnson, J.: Children, robotics, and education. Artificial Life and Robotics 7(1-2), 16-21 (mar 2003)

6. Kolarevic, B.: Digital Fabrication : Manufacturing Architecture in the Information Age. Proceedings of the Twenty First Annual Conference of the Association for Computer-Aided Design in Architecture / ISBN 1-880250-10-1 pp. 268-278 (2001)

7. Lerner, J., Tirole, J.: The open source movement: Key research questions. European Economic Review 45(4-6), 819-826 (2001)

8. Mostert-Van Der Sar, M., Mulder, I., Remijn, L., Troxler, P.: FabLabs in design education. Proceedings of the 15th International Conference on Engineering and Product Design Education: Design Education - Growing Our Future, EPDE 2013 (September), 629-634 (2013)

9. Sabanovic, S., Michalowski, M.P., Simmons, R.: Robots in the wild: Observing human-robot social interaction outside the lab. In: International Workshop on Advanced Motion Control, AMC. vol. 2006, pp. 576-581 (2006)

10. Vandevelde, C., Saldien, J.: An Open Platform for the Design of Social Robot Embodiments for Face-to-Face Communication. In: Proceedings of the 11th International Conference on Human-Robot Interaction. pp. 287-294. Christchurch, New Zealand (2016)

11. Vandevelde, C., Vanhoucke, M., Saldien, J.: Prototyping Social Interactions with DIY Animatronic Creatures. In: Proceedings of the Ninth International Conference on Tangible, Embedded, and Embodied Interaction. pp. 509-512. TEI '15, ACM, New York, NY, USA (2015)

12. Viola, P., Jones, M.: Robust real-time face detection. International Journal of Computer Vision 57(2), 137-154 (2004), http://link.springer.com/article/10.1023/B:VISI.0000013087.49260.fb

13. de Wit, C.: Trends in mobile robot and vehicle control. In: Siciliano, B., Valavanis, K.P. (eds.) Control Problems in Robotics and Automation, pp. 151-175. Springer Berlin Heidelberg, Berlin, Heidelberg (1998) 